\title{
Relativistic corrections to the quark-antiquark potential from the effective string theory
}

\section{Hector E. Martinez*}

Physik-Department Technische Universität München

E-mail: hector.martinez@tum.de

\begin{abstract}
We use the complete expression for the $\mathscr{O}\left(1 / \mathrm{m}^{2}\right)$ corrections to the quark-antiquark potential derived from QCD in terms of Wilson loop expectation values, and a mapping, valid at large distances, between those Wilson loop expectation values and correlators evaluated in the effective string theory, to compute all $\mathscr{O}\left(1 / \mathrm{m}^{2}\right)$ potentials at large distances. In particular, we present previously unknown results for the spin-independent part of the potential and confirm known results for the spin and momentum dependent parts. Finally, we calculate the relativistic corrections induced by the newly calculated potentials to the string spectrum.
\end{abstract}

Xth Quark Confinement and the Hadron Spectrum

8 - 12 October 2012

TUM Campus Garching, Munich, Germany

\footnotetext{
* Speaker.
} 


\section{Introduction}

Theoretical understanding of the heavy quarkonium phenomenology is one of the major challenges in current QCD research. In the past we have relied on potential models that turned out to be very successful explaining heavy quarkonium phenomenology but which could not be derived directly from QCD. In more recent years effective field theories (EFTs) like non-relativistic QCD (NRQCD) [1, 2] and potential non-relativistic QCD (pNRQCD) [3, 4] have accounted for this problem exploiting the scale hierarchy inherited from the non-relativistic nature of the bound system. NQRCD is obtained when the degrees of freedom with energies of the order of the hard scale are integrated out. pNRQCD is obtained from NRQCD by integrating out modes of energy of the soft scale. The resulting theory is close to a quantum mechanical description of the bound system where the matching coefficients appear as potentials that may be expanded in powers of $1 / \mathrm{m}$. We shall distinguish between strongly coupled quarkonia, for which the relative momentum of the quarkantiquark pair is the order of $\Lambda_{Q C D}$ (the typical hadronic scale) and weakly coupled quarkonia, for which the relative momentum is much higher than $\Lambda_{Q C D}$. In the first case, the matching has to be done in a non-perturbative fashion, then the degrees of freedom of pNRQCD are singlet quarkonium fields. For the weak-coupling case, the matching may be done order by order in $\alpha_{s}$, in this case the degrees of freedom of the theory include also octet quarkonium fields and ultrasoft gluons which are multiple expanded about the center-of-mass coordinate. This picture could remind us the old potential models, however, in contrast with them, pNRQCD is equivalent with QCD when evaluated at the same kinematic regime. In the framework of pNRQCD the relativistic corrections to the quark-antiquark potential have been obtained in terms of operator insertions in the expectation value of the rectangular Wilson loop $[5,6]$. These expressions are valid beyond perturbation theory and hold also in the long-distance regime. In this work we complete the calculation started in [7] in which these expressions are calculated in the effective string theory (EST). This theory states that the interaction between quark and antiquark in the long-distance regime can be described through the string action. We complete that calculation to get the full $1 / \mathrm{m}^{2}$ suppressed potential in the string description.

This paper is organized as follows, the section 2 we will show how the $\mathscr{O}(1 / m)$ and $\mathscr{O}\left(1 / \mathrm{m}^{2}\right)$ corrections are organized, in section 3 we introduce the EST and present the mapping and string correlator that we will use to compute the potential corrections in the EST, section 4 show our results for the full $\mathscr{O}\left(1 / \mathrm{m}^{2}\right)$ corrections in the string description, in section 5 we use these corrections to construct the string spectrum in a simple model, finally our summary is presented in section 6 .

\section{Relativistic corrections to the quarkonium potential}

In order to make our results clear we will organize the corrections to the quarkonium potential as they were presented in [6]. Up to $1 / \mathrm{m}^{2}$ the Hamiltonian for two quarks is given by

$$
H=\frac{\mathbf{p}_{1}^{2}}{2 m_{1}}+\frac{\mathbf{p}_{2}^{2}}{2 m_{2}}+V^{(0)}+V^{(1 / m)}+V^{\left(1 / m^{2}\right)}
$$

where the static potential is given by

$$
V^{(0)}(r)=\lim _{T, T_{W} \rightarrow \infty} \frac{i}{T}\left\langle W_{\square}\right\rangle
$$


and $\left\langle W_{\square}\right\rangle$ is the expected value of the rectangular Wilson loop

$$
W_{\square} \equiv \operatorname{Pexp}\left\{-i g \oint_{r \times T_{W}} d z^{\mu} A_{\mu}(z)\right\},
$$

with P being the path-ordering operator. We write the $1 / m$ and $1 / m^{2}$ suppressed corrections as

$$
\begin{aligned}
V^{(1 / m)} & =\frac{V^{(1,0)}}{m_{1}}+\frac{V^{(0,1)}}{m_{2}}, \\
V^{\left(1 / m^{2}\right)} & =\frac{V^{(2,0)}}{m_{1}^{2}}+\frac{V^{(0,2)}}{m_{2}^{2}}+\frac{V^{(1,1)}}{m_{1} m_{2}},
\end{aligned}
$$

where mass exchange invariance implies $V^{(1,0)}=V^{(0,1)}$. It is customary to separate the contributions between spin-dependent and spin-independent parts

$$
\begin{aligned}
& V^{(2,0)}=V_{S D}^{(2,0)}+V_{S I}^{(2,0)}, \\
& V^{(0,2)}=V_{S D}^{(0,2)}+V_{S I}^{(0,2)},
\end{aligned}
$$

where

$$
\begin{aligned}
& V_{S I}^{(2,0)}=\frac{1}{2}\left\{\mathbf{p}_{1}, V_{p^{2}}^{(2,0)}(r)\right\}+\frac{V_{L^{2}}^{(2,0)}(r)}{r^{2}} \mathbf{L}_{1}^{2}+V_{r}^{(2,0)}(r), \\
& V_{S I}^{(0,2)}=\frac{1}{2}\left\{\mathbf{p}_{2}, V_{p^{2}}^{(0,2)}(r)\right\}+\frac{V_{L^{2}}^{(0,2)}(r)}{r^{2}} \mathbf{L}_{2}^{2}+V_{r}^{(0,2)}(r),
\end{aligned}
$$

$\mathbf{p}_{1}=-i \nabla_{x_{1}}$ and $\mathbf{L}_{1}=\mathbf{r} \times \mathbf{p}_{1}$. Invariance under charge conjugation and mass interchange yields

$$
\begin{gathered}
V_{p^{2}}^{(2,0)}(r)=V_{p^{2}}^{(0,2)}(r), \\
V_{L^{2}}^{(2,0)}(r)=V_{L^{2}}^{(0,2)}(r), \\
V_{r}^{(2,0)}(r)=V_{r}^{(0,2)}(r) .
\end{gathered}
$$

For the spin-dependent part we have

$$
\begin{aligned}
& V_{S D}^{(2,0)}=V_{L S}^{(2,0)}(r) \mathbf{L}_{1} \cdot \mathbf{S}_{1}, \\
& V_{S D}^{(0,2)}=-V_{L S}^{(0,2)}(r) \mathbf{L}_{2} \cdot \mathbf{S}_{2} .
\end{aligned}
$$

Charge conjugation and mass exchange invariance implies $V_{L S}^{(2,0)}=V_{L S}^{(0,2)}$. For the $V^{(1,1)}$ potential we make the same decomposition

$$
V^{(1,1)}=V_{S D}^{(1,1)}+V_{S I}^{(1,1)}
$$

where

$$
V_{S I}^{(1,1)}=-\frac{1}{2}\left\{\mathbf{p}_{\mathbf{1}} \cdot \mathbf{p}_{2}, V_{p^{2}}^{(1,1)}(r)\right\}-\frac{V_{L^{2}}^{(1,1)}(r)}{2 r^{2}}\left(\mathbf{L}_{\mathbf{1}} \cdot \mathbf{L}_{\mathbf{2}}+\mathbf{L}_{\mathbf{2}} \cdot \mathbf{L}_{\mathbf{1}}\right)+V_{r}^{(1,1)}(r)
$$

and

$$
V_{S D}^{(1,1)}=V_{L_{1} S_{2}}^{(1,1)}(r) \mathbf{L}_{\mathbf{1}} \cdot \mathbf{S}_{\mathbf{2}}-V_{L_{2} S_{1}}^{(1,1)}(r) \mathbf{L}_{\mathbf{2}} \cdot \mathbf{S}_{\mathbf{1}}+V_{S^{2}}^{(1,1)}(r) \mathbf{S}_{\mathbf{1}} \cdot \mathbf{S}_{\mathbf{2}}+V_{S_{12}}^{(1,1)}(r) \mathbf{S}_{\mathbf{1 2}}(\hat{\mathbf{r}})
$$


with

$$
\mathbf{S}_{12}(\hat{\mathbf{r}})=3 \hat{\mathbf{r}} \cdot \sigma_{1} \hat{\mathbf{r}} \cdot \sigma_{2}-\sigma_{1} \cdot \sigma_{2}
$$

where charge conjugation and mass exchange invariance implies $V_{L_{1} S_{2}}^{(1,1)}=V_{L_{2} S_{1}}^{(1,1)}$.

The $1 / m$ and $1 / m^{2}$ suppressed corrections $V^{(1,0)}, V^{(2,0)}, V^{(0,2)}$ and $V^{(1,1)}$ have been derived from QCD in terms of operator insertions in the Wilson loop expectation value [5, 6]. These expressions were obtained from the matching between NRQCD and pNRQCD up to $1 / \mathrm{m}^{2}$ in the relativistic expansion and hold beyond perturbation theory in the long-distance regime. For instance, the $1 / \mathrm{m}$ suppressed correction obtained in [5] reads

$$
V^{(1,0)}=-\frac{g^{2}}{2} \lim _{T, T_{W} \rightarrow \infty} \int_{0}^{T} d t t\left\{\frac{\left\langle 0\left|\mathbf{E}_{1}(t) \mathbf{E}_{2}(0) W_{\square}\right| 0\right\rangle}{\left\langle 0\left|W_{\square}\right| 0\right\rangle}-\frac{\left\langle 0\left|\mathbf{E}_{1}(t) W_{\square}\right| 0\right\rangle\left\langle 0\left|\mathbf{E}_{2}(0) W_{\square}\right| 0\right\rangle}{\left\langle 0\left|W_{\square}\right| 0\right\rangle^{2}}\right\} .
$$

The $1 / \mathrm{m}^{2}$ suppressed corrections follow the same structure but due to space constraints we refer the reader to [6] for explicit formulas.

\section{The effective string theory}

The linear dependence in $r$ of the static potential (2.2), derived from the expectation value of the rectangular Wilson loop, suggests that at long distances the quark antiquark interaction could be described by a string. The effective string theory (EST) states that in the long-distance regime $\left(r \Lambda_{Q C D} \gg 1\right)$ the expectation value of the rectangular Wilson loop can be obtained from the string action

$$
\lim _{T_{W} \rightarrow \infty}\left\langle 0\left|W_{\square}\left(T_{W}, r\right)\right| 0\right\rangle=Z \int \mathscr{D} \xi^{1} \mathscr{D} \xi^{2} e^{i S_{\text {string }}\left(\xi^{1}, \xi^{2}\right)}
$$

where $Z$ is an unknown constant, the string action is given by

$$
S_{\text {string }}=\int d t d z \mathscr{L}\left(\partial^{\mu} \xi^{l}\right)=-\sigma \int d t d z\left(1-\frac{1}{2} \partial_{\mu} \xi^{l} \partial^{\mu} \xi^{l}\right)
$$

$\sigma$ is the string tension and $\xi^{l}=\xi^{l}(t, z)(l=1,2)$ are the transverse components of the string.

The EST hypothesis gives the following prediction for the long-distance behavior of the static potential [8]

$$
V^{(0)}(r)=\sigma r+\mu-\frac{\pi}{12 r}
$$

where $\mu$ is an unknown constant with dimension of mass.

Since both are valid on the long-distance regime, one could expect to have a description in the EST of the potential corrections obtained in [5] and [6]. Requiring the same symmetry (C, P, T) properties for the transverse string coordinates and for the Wilson loop operator insertions, the following mapping into the EST for the operator insertions has been obtained [7, 9]

$$
\begin{aligned}
\psi^{\dagger}(t) \mathbf{E}^{l}\left(t, \frac{\mathbf{r}}{2}\right) \psi(t) & \mapsto \Lambda^{2} \partial_{z} \xi^{l}\left(t, \frac{r}{2}\right) \\
\chi^{\dagger}(t) \mathbf{E}^{l}\left(t,-\frac{\mathbf{r}}{2}\right) \chi(t) & \mapsto-\Lambda^{2} \partial_{z} \xi^{l}\left(t,-\frac{r}{2}\right) \\
\psi^{\dagger}(t) \mathbf{B}^{l}\left(t, \frac{\mathbf{r}}{2}\right) \psi(t) & \mapsto \Lambda^{\prime} \varepsilon^{l m} \partial_{t} \partial_{z} \xi^{m}\left(t, \frac{r}{2}\right)
\end{aligned}
$$




$$
\begin{aligned}
\chi^{\dagger}(t) \mathbf{B}^{l}\left(t,-\frac{\mathbf{r}}{2}\right) \chi(t) & \mapsto \Lambda^{\prime} \varepsilon^{l m} \partial_{t} \partial_{z} \xi^{l}\left(t,-\frac{r}{2}\right) \\
\psi^{\dagger}(t) \mathbf{E}^{3}\left(t, \frac{\mathbf{r}}{2}\right) \psi(t) & \mapsto \Lambda^{\prime \prime 2} \\
\chi^{\dagger}(t) \mathbf{E}^{3}\left(t,-\frac{\mathbf{r}}{2}\right) \chi(t) & \mapsto-\Lambda^{\prime \prime 2} \\
\psi^{\dagger}(t) \mathbf{B}^{3}\left(t, \frac{\mathbf{r}}{2}\right) \psi(t) & \mapsto \Lambda^{\prime \prime \prime} \varepsilon^{l m} \partial_{t} \partial_{z} \xi^{l}\left(t, \frac{r}{2}\right) \partial_{z} \xi^{m}\left(t, \frac{r}{2}\right) \\
\psi^{\dagger}(t) \mathbf{B}^{3}\left(t,-\frac{\mathbf{r}}{2}\right) \psi(t) & \mapsto \Lambda^{\prime \prime \prime} \varepsilon^{l m} \partial_{t} \partial_{z} \xi^{l}\left(t,-\frac{r}{2}\right) \partial_{z} \xi^{m}\left(t,-\frac{r}{2}\right) .
\end{aligned}
$$

where $\Lambda, \Lambda^{\prime}, \Lambda^{\prime \prime}, \Lambda^{\prime \prime \prime} \sim \Lambda_{Q C D}$ are unknown constants with dimension of mass and $l, m=1,2$.

Using this mapping we will be able to calculate expressions for the potentials in the EST that will depend on the two-coordinates string correlator $\left\langle\xi^{l}(t, z) \xi^{m}\left(t^{\prime}, z^{\prime}\right)\right\rangle$ which, in the Euclidean space, is given by $[10,11]$

$$
G_{F}^{l m}\left(i t, z ; i t^{\prime}, z^{\prime}\right)=\frac{\delta^{l m}}{4 \pi \sigma} \ln \left(\frac{\cosh \left(\frac{\pi}{r}\left(t-t^{\prime}\right)\right)+\cos \left(\frac{\pi}{r}\left(z+z^{\prime}\right)\right)}{\cosh \left(\frac{\pi}{r}\left(t-t^{\prime}\right)\right)-\cos \left(\frac{\pi}{r}\left(z-z^{\prime}\right)\right)}\right) .
$$

\section{Potential corrections in the EST}

Provided with the mapping and the correlator we proceed to compute the potential correction in the EST. For instance, in the case of the $V^{(1,0)}$ correction given previously the EST description that follows from the mapping reads

$$
\begin{aligned}
\frac{\left\langle 0\left|\mathbf{E}_{1}(t) \mathbf{E}_{2}(0) W_{\square}\right| 0\right\rangle}{\left\langle 0\left|W_{\square}\right| 0\right\rangle}-\frac{\left\langle 0\left|\mathbf{E}_{1}(t) W_{\square}\right| 0\right\rangle\left\langle 0\left|\mathbf{E}_{2}(0) W_{\square}\right| 0\right\rangle}{\left\langle 0\left|W_{\square}\right| 0\right\rangle^{2}} & \mapsto \Lambda^{4} \partial_{z} \partial_{z^{\prime}}\left\langle\xi^{l}(t, r / 2) \xi^{m}(0, r / 2)\right\rangle \\
& =\Lambda^{4} \partial_{z} \partial_{z^{\prime}} G_{F}^{l m}(t, r / 2 ; 0, r / 2) \\
& =-\frac{\delta_{l m}}{4 \kappa} \frac{\pi}{r^{2} \sin ^{2}\left(\frac{\pi t}{2 r}\right)} .
\end{aligned}
$$

Integrating we get

$$
V^{(1,0)}(r)=\frac{g^{2} \Lambda^{4}}{\sigma \pi} \ln (\sqrt{\sigma} r)-\ln \left(\varepsilon_{U V}\right)
$$

where we have introduced a UV cutoff for small times in order to regularize the integral, this result agrees with [7].

Following the same procedure for the previously unknown corrections we get

$$
\begin{aligned}
& V_{r}^{(2,0)}(r)=-\frac{2 g^{4} \Lambda^{8} r}{\sigma^{2} \pi^{3}} I^{(2,0)}-\frac{g^{2} c_{F}^{(1) 2} \Lambda^{\prime 2}}{\sigma \pi \varepsilon_{U V}^{3}}+\frac{3 g^{2} \Lambda^{4}}{2 \pi^{3} \sigma} \zeta(3)\left(\nabla_{r}^{i} r^{2} \nabla_{r}^{i} V^{(0)}\right)-\frac{g^{2} \Lambda^{4} r^{3}}{45 \sigma}\left(\nabla_{r}^{i} V^{(0)}\right)\left(\nabla_{r}^{i} V^{(0)}\right) \\
& V_{r}^{(1,1)}(r)=-\frac{4 g^{4} \Lambda^{8} r}{\sigma^{2} \pi^{3}} I^{(1,1)}-\frac{9 g^{2} \Lambda^{4} \zeta(3)}{4 \pi^{3} \sigma} \nabla_{r}^{i}\left(r^{2} \nabla_{r}^{i} V^{(0)}\right)+\frac{7 g^{2} \Lambda^{4} r^{3}}{180 \sigma}\left(\nabla_{r}^{i} V^{(0)}\right)\left(\nabla_{r}^{i} V^{(0)}\right) \\
& V_{S^{2}}^{(1,1)}(r)=\frac{2 g^{2} c_{F}^{(1)} c_{F}^{(2)} \Lambda^{\prime \prime \prime} \pi^{3}}{45 \sigma^{4} r^{5}} \\
& V_{S_{12}}^{(1,1)}(r)=\frac{1}{4} V_{S^{2}}^{(1,1)}
\end{aligned}
$$


where $c_{F}^{(1)}, c_{F}^{(2)}$ are coefficients coming from the matching between NRQCD and QCD and

$$
\begin{aligned}
& I^{(2,0)}=\int_{0}^{\infty} d t_{1} \int_{0}^{t_{1}} d t_{2} \int_{0}^{t_{2}} d t_{3}\left(t_{2}-t_{3}\right)^{2}\left\{\frac{1}{\sinh ^{2}\left(t_{2}\right) \sinh ^{2}\left(t_{1}-t_{3}\right)}+\frac{1}{\sinh ^{2}\left(t_{1}\right) \sinh ^{2}\left(t_{2}-t_{3}\right)}\right\} \\
& I^{(1,1)}=\int_{0}^{\infty} d t_{1} \int_{0}^{t_{1}} d t_{2} \int_{0}^{t_{2}} d t_{3}\left(t_{2}-t_{3}\right)^{2}\left\{\frac{1}{\cosh ^{2}\left(t_{2}\right) \cosh ^{2}\left(t_{1}-t_{3}\right)}+\frac{1}{\cosh ^{2}\left(t_{1}\right) \cosh ^{2}\left(t_{2}-t_{3}\right)}\right\} .
\end{aligned}
$$

As we did for $V^{(1,0)}$ we have introduced a new UV cutoff in order to regularize $V_{r}^{(2,0)}$. In both cases the effect of these cutoffs is to add an infinite constant, we renormalize the potentials adding these constants to the unknown $\mu$ parameter appearing in (3.3).

The remaining potentials have been already calculated in [7], our results for the renormalized potentials agree with theirs:

$$
\begin{aligned}
V_{p^{2}}^{(1,1)}(r) & =0 \\
V_{p^{2}}^{(2,0)}(r) & =0 \\
V_{L^{2}}^{(2,0)}(r) & =-\frac{g^{2} \Lambda^{4} r}{6 \sigma}, \\
V_{L^{2}}^{(1,1)}(r) & =\frac{g^{2} \Lambda^{4} r}{6 \sigma} \\
V_{L S}^{(2,0)}(r) & =-\frac{\mu_{c}}{r}-\frac{g^{2} c_{F}^{(1)} \Lambda^{\prime} \Lambda^{2}}{\sigma r^{2}}+\frac{c_{S}^{(1)} \pi}{24 r^{3}} \\
V_{L_{2} S_{1}}^{(1,1)}(r) & =-\frac{g^{2} c_{F}^{(1)} \Lambda^{2} \Lambda^{\prime}}{\sigma r^{2}}, \\
V_{L_{1} S_{2}}^{(1,1)}(r) & =-\frac{g^{2} c_{F}^{(1)} \Lambda^{2} \Lambda^{\prime}}{\sigma r^{2}}
\end{aligned}
$$

where $c_{S}^{(1)}$ is another coefficient coming from the matching between NRQCD and QCD and $\mu_{c}$ is a new, in principle undetermined, constant coming from the regularization of the $V_{L S}^{(2,0)}$ potential. In this case we can not directly apply the renormalization procedure we applied for $V^{(1,0)}$ and $V_{r}^{(2,0)}$ because the divergent integral is now proportional to $1 / r$, instead we notice the argument pointed out in [7] that this divergence behavior is not a problem of the EST itself but one inherited from QCD and that for regularize this potential one has to include in the mapping also the counter terms that regularize the UV divergences. In this way the constant $\mu_{c}$ we have introduced will depend on the finite part of these counter terms once they are included.

For details of the calculation of the corrections we refer the reader to [11].

The calculated corrections are constrained by Poincaré invariance, constraint that translates to the potentials as the Gromes relation [12] and the first Brambilla-Barchielli-Montaldi-Prosperi relation [13]

$$
\begin{aligned}
& \frac{r}{2} \frac{d V^{(0)}}{d r}+2 V_{L^{2}}^{(2,0)}-V_{L^{2}}^{(1,1)}=0 \\
& \frac{1}{2 r} \frac{d V^{(0)}}{d r}+V_{L S}^{(2,0)}-V_{L_{2} S_{1}}^{(1,1)}=0
\end{aligned}
$$


which leads to

$$
\begin{aligned}
\mu_{c} & =\sigma / 2 \\
g \Lambda^{2} & =\sigma,
\end{aligned}
$$

in this way our unknown constant $\mu_{c}$ coming from the renormalization of the $V_{L S}^{(2,0)}$ potential is now fixed in terms of the string tension $\sigma$.

\section{The Spectrum}

To show an application of our results we compute the spectrum defined by the $\mathscr{O}\left(1 / \mathrm{m}^{2}\right)$ potential in a simple model in which only the leading corrections up to $1 / r$ are taken in to account. With this criteria potentials like $V_{S^{2}}^{(1,1)}$ do not contribute since it scales in $r$ like $1 / r^{5}$. Applying this rule to the other corrections we get

$$
\begin{aligned}
V^{(1,0)}(r) & =\frac{\sigma}{\pi} \ln (\sqrt{\sigma} r), \\
V_{L^{2}}^{(2,0)}(r) & =-\frac{\sigma r}{6}, \\
V_{L^{2}}^{(1,1)}(r) & =\frac{\sigma r}{6}, \\
V_{L S}^{(2,0)}(r) & =-\frac{\sigma}{2 r}, \\
V_{r}^{(2,0)}(r) & =0.155 \sigma^{2} r-0.044 \sigma^{3} r^{3}, \\
V_{r}^{(1,1)}(r) & =-0.368 \sigma^{2} r+0.078 \sigma^{3} r^{3},
\end{aligned}
$$

where we have calculated the derivatives, used (4.16), (4.17) and numerically calculated the integrals $I^{(2,0)}$ and $I^{(1,1)}$ in order get compact expressions. All the other potentials will not contribute. In the case of $V^{(0)}$ we will only consider the linear part (proportional to $\sigma$ ) and we will restrict ourselves to the equal mass case. The resulting potential for the model is given by

$$
\begin{aligned}
V(r) & =\sigma r+\frac{2}{m} V^{(1,0)}+\frac{1}{m^{2}}\left\{\frac{V_{L^{2}}^{(2,0)}}{r^{2}} \mathbf{L}^{2}+V_{L S}^{(2,0)} \mathbf{L} \cdot \mathbf{S}+2 V_{r}^{(2,0)}+V_{r}^{(1,1)}\right\} \\
& =\sigma r+\frac{1}{m}\left\{\frac{2 \sigma}{\pi} \ln (\sqrt{\sigma} r)\right\}+\frac{1}{m^{2}}\left\{-\frac{\sigma}{6 r} \mathbf{L}^{2}-\frac{\sigma}{2 r} \mathbf{L} \cdot \mathbf{S}-0.058 \sigma^{2} r-0.011 \sigma^{3} r^{3}\right\}
\end{aligned}
$$

notice that this potential only depend on $\sigma$ and $m$.

To compute the spectrum we will consider the $\mathscr{O}(1 / m)$ and $\mathscr{O}\left(1 / m^{2}\right)$ corrections as perturbations, to be consistent with the counting we have to go up to second order in perturbation theory for the $1 / m$ correction, however, this correction does not give any additional insight since it just add a constant $\left(1 / \mathrm{m}^{2}\right.$ suppressed) shift to the spectral lines, to make the calculation simpler we will not consider it. In Fig. (1) we show part of the spectrum for different values of $m$ for a fixed value of $\sigma$. 


\section{String Spectrum for $m=10,11,12$}

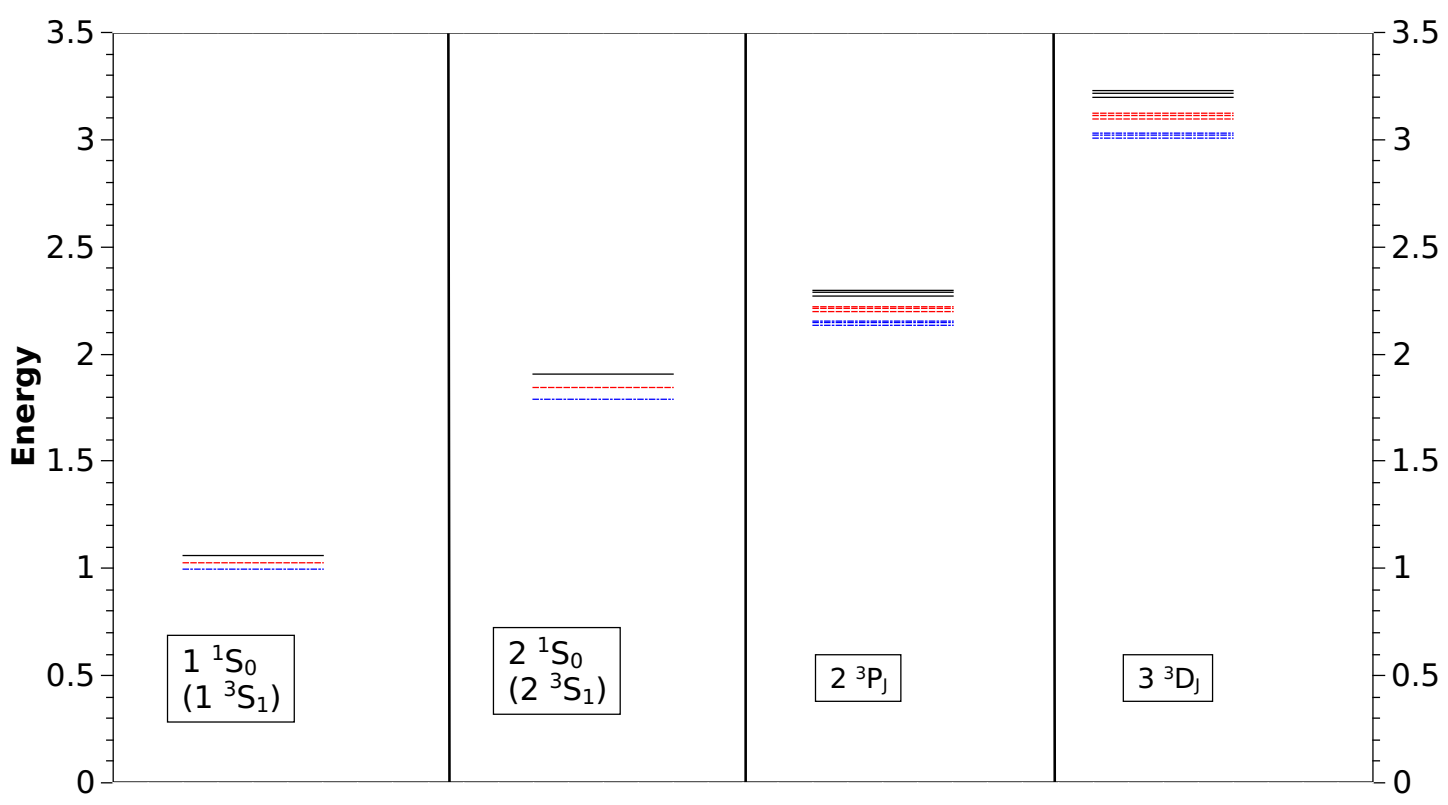

Figure 1: Energy levels of some characteristic angular momentum states for the first three principal quantum numbers in the model. We set $\sqrt{\sigma}=1$ so the energy is in units of $\sigma$. Black: $m=10$, red: $m=11$, blue: $m=12$. The $1 / m^{2}$ suppression of the $\mathbf{L}^{2}$ and $\mathbf{L} \cdot \mathbf{S}$ corrections make the line splitting very small.

\section{Summary}

We have calculated the complete $\mathscr{O}\left(1 / \mathrm{m}^{2}\right)$ quarkonium potential in the EST, in particular we have obtained previously unknown results for the spin independent part. As an application of our results we have computed the spectrum defined by a model in in which only the leading parts in $r$ are considered. Recently the spin-orbit $1 / \mathrm{m}^{2}$ suppressed potential has been extracted from the lattice [14], we expect that when more of the $1 / \mathrm{m}^{2}$ potentials become available the EST will provide an economical way to parametrize lattice results.

\section{Acknowledgements}

This work was done in collaboration with Nora Brambilla and Antonio Vairo from the TU München and Michael Groher from the ETH Zürich. The author acknowledge financial support from DAAD.

\section{References}

[1] W. E. Caswell and G. P. Lepage, Phys. Lett. B 167, 437 (1986).

[2] G. T. Bodwin, E. Braaten and G. P. Lepage, Phys. Rev. D 51, 1125 (1995) [Erratum-ibid. D 55, 5853 (1997)] [hep-ph/9407339]. 
[3] A. Pineda and J. Soto, Nucl. Phys. Proc. Suppl. 64, 428 (1998) [hep-ph/9707481].

[4] N. Brambilla, A. Pineda, J. Soto and A. Vairo, Nucl. Phys. B 566, 275 (2000) [hep-ph/9907240].

[5] N. Brambilla, A. Pineda, J. Soto and A. Vairo, Phys. Rev. D 63, 014023 (2001) [hep-ph/0002250].

[6] A. Pineda and A. Vairo, Phys. Rev. D 63, 054007 (2001) [Erratum-ibid. D 64, 039902 (2001)] [hep-ph/0009145].

[7] G. Perez-Nadal and J. Soto, Phys. Rev. D 79, 114002 (2009) [arXiv:0811.2762 [hep-ph]].

[8] M. Luscher, K. Symanzik and P. Weisz, Nucl. Phys. B 173, 365 (1980).

[9] J. B. Kogut and G. Parisi, Phys. Rev. Lett. 47, 1089 (1981).

[10] Private communication between Perez-Nadal Antonio Vairo and Nora Brambilla.

[11] N. Brambilla, M. Groher, H. E. Martinez, A. Vairo TUM-EFT 36/12.

[12] D. Gromes, Z. Phys. C 26, 401 (1984).

[13] A. Barchielli, N. Brambilla and G. M. Prosperi, Nuovo Cim. A 103, 59 (1990).

[14] Y. Koma and M. Koma, PoS LATTICE 2012, 140 (2012) [arXiv:1211.6795 [hep-lat]]. 